\title{
Early signatures of large-scale field line opening
}

\section{Multi-wavelength analysis of features connected with a "halo" CME event}

\author{
S. Pohjolainen ${ }^{1}$, N. Vilmer ${ }^{2}$, J. I. Khan ${ }^{3}$, and A. E. Hillaris ${ }^{4}$ \\ 1 Tuorla Observatory/Department of Physics, University of Turku, 21500 Piikkiö, Finland \\ e-mail: silpoh@utu.fi \\ 2 LESIA, UMR CNRS 8109, Observatoire de Paris, 5 place J. Janssen, 92195 Meudon, France \\ e-mail: nicole.vilmer@obspm.fr \\ 3 Dept. of Physics \& Astronomy, University of Glasgow, Glasgow G12 8QQ, Scotland, UK \\ e-mail: jkhan@spd.aas.org \\ 4 Section of Astrophysics, Astronomy and Mechanics, Department of Physics, University of Athens, 15784 Panepistimiopolis \\ Zografos, Athens, Greece \\ e-mail: ahilaris@cc.uoa.gr
}

Received 31 May 2004 / Accepted 13 December 2004

\begin{abstract}
A fast "halo"-type coronal mass ejection (CME) associated with a two-ribbon flare, GOES class M 1.3, was observed on February 8, 2000. Soft X-ray and EUV images revealed several loop ejections and one wave-like moving front that started from a remote location, away from the flare core region. A radio type-II burst was observed near the trajectory of the moving soft X-ray front, although association with the CME itself cannot be ruled out. Large-scale dimmings were observed in EUV and soft X-rays, both in the form of disappearing transequatorial loops. We can pinpoint the time and the location of the first large-scale field-line opening by tracing the electron propagation paths above the active region and along the transequatorial loop system, in which large-scale mass depletion later took place. The immediate start of a type-IV burst (interpreted as an upward moving structure) which was located over a soft X-ray dimming region, confirms that the CME had lifted off. We compare these signatures with those of another halo CME event observed on May 2, 1998, and discuss the possible connections with the "magnetic breakout" model.
\end{abstract}

Key words. Sun: flares - shock waves - Sun: coronal mass ejections

\section{Introduction}

"Halo" coronal mass ejections (CMEs) that originate on the visible solar hemisphere can often be connected with signatures of eruptions and large scale changes seen on the solar disk. Halo CMEs have been associated with EUV and soft $\mathrm{X}$-ray dimmings, disappearing transequatorial loops, as well as Moreton and EIT waves. EUV and soft X-ray dimmings (Sterling \& Hudson 1997; Zarro et al. 1999; Thompson et al. 2000). Disappearing transequatorial loops (Khan \& Hudson 2000; Pohjolainen et al. 2001) represent depleted matter, while $\mathrm{H} \alpha$ (Moreton) waves and EIT waves have mostly been connected with flares (Warmuth et al. 2001; Hudson et al. 2003).

Flares and CMEs are often associated with decimetricmetric radio emission that originate at coronal heights: type III radio bursts are produced by plasma emission processes when a stream of mildly relativistic electrons travel along open magnetic field lines (Vlahos \& Raoult 1995; Bastian et al. 1998). The U- and J-subtypes of the type III bursts result from energetic electrons streaming along long but closed loops, and they thus exhibit a characteristic turn-over frequency at the tops of the loops (Aurass \& Klein 1997). Short radio type III bursts that mark the initial instability in a small volume before large scale changes have previously been reported by Maia et al. (1998, 1999) and Aurass et al. (1999).

Type II radio bursts at decimetric-metric wavelengths result, ultimately, from plasma emission at MHD shock fronts (Wild \& Smerd 1972) and they can thus trace the propagation paths of both plasma ejecta and shock waves (Klein et al. 1999, 2003). Therefore close connections with CMEs (piston-driven shock fronts by the leading edges or internal parts of the CME) and flares (blast waves or flare ejecta acting as piston) have been established (Maia et al. 2000; Claßen \& Aurass 2002; Hudson et al. 2003).

Type IV radio emission most often occurs in conjunction with type II emission and has a close association with CMEs (Kahler 1992). It can be stationary or moving (see, e.g., Kundu 1965; Gopalswamy \& Kundu 1990), and it can be either plasma emission or gyrosynchrotron emission, produced by particles trapped in a magnetic cloud (Bastian et al. 2001). A number 


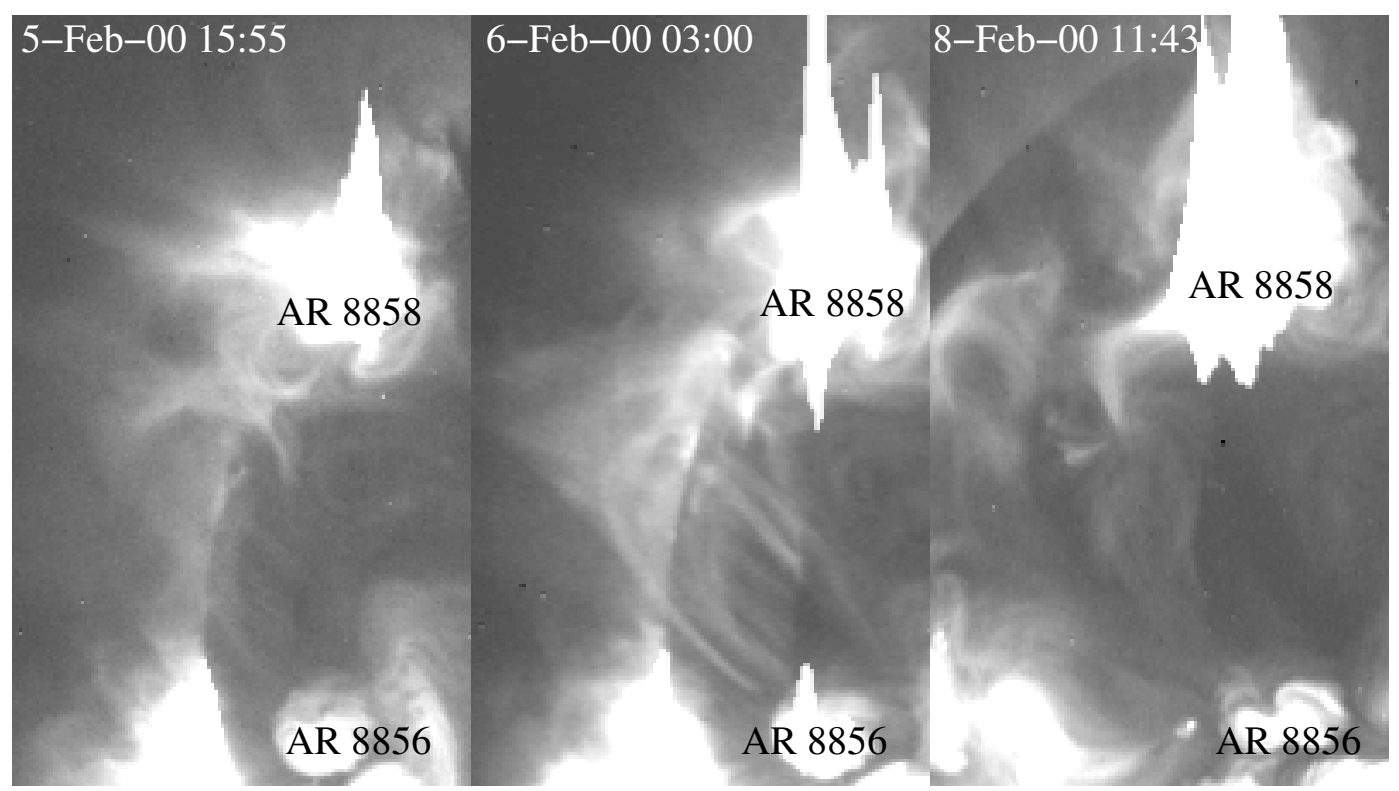

Fig. 1. Yohkoh SXT images showing the X-ray connectivities on February 5, 6, and 8, 2000. The first two images show the complex loop structures, with possible coronal X-points and transequatorial loops connecting the two active regions NOAA 8858 and 8856 . The last image on February 8 is post-flare and post-CME, when some of the loops have already disappeared.

of type IV bursts are believed to originate within the dense substructures of CMEs (Aurass et al. 1999) and they have been associated with erupting filaments within CMEs (Klein $\&$ Mouradian 2002).

Our interest in this paper is to study the onset of CMErelated solar eruptions by analysing one event in detail, for which ample multiwavelength observations are available. The event on February 8, 2000 was reported to include a fast "halo" CME, a flare, an EUV wave and a dimming, and radio type II, III and IV emission. On closer inspection, it also included a transequatorial soft $\mathrm{X}$-ray loop that underwent similar dimming as the EUV counterparts.

We present a multiwavelength analysis of the signatures closely related with the large-scale destabilization of the field and give interpretations for the multiple ejections and radio bursts observed during the event. In the last section we also compare the February 8, 2000 event with a similar event observed on May 2, 1998 (Pohjolainen et al. 2001).

\section{Observations}

\subsection{Instrumentation}

The February 8, 2000 event was observed by SOHO LASCO (Large Angle Spectrometric Coronagraph, Brueckner et al. 1995) that images the corona in white light, by SOHO EIT in $195 \AA$ with 12 min image cadence (EUV Imaging Telescope, Delaboudinière et al. 1995), and by TRACE in $171 \AA$ A with 45 s image cadence (Transition Region and Coronal Explorer, Handy at al. 1999). The Fe XII line (195 $\AA)$ is formed around $1.5 \mathrm{MK}$ and the Fe IX/X (171 $\AA$ ) around 1.3 MK temperature. SOHO MDI (Michelson Doppler Imager, Scherrer et al. 1995) mapped the longitudinal magnetic flux density averaged over the pixel field of view. Yohkoh SXT (Soft X-Ray Telescope, Tsuneta et al. 1991) imaged the event in soft X-rays, and the
Yohkoh HXT (Hard X-Ray Telescope, Kosugi et al. 1991) in hard X-rays. Yohkoh SXT records coronal temperatures in the 2-20 MK range. All the difference images have been corrected for solar rotation.

Radio spectral data were obtained from the ARTEMIS-IV spectrograph covering the spectral range $110-680 \mathrm{MHz}$ (Caroubalos et al. 2000), from the Tremsdorf OSRA spectrographs working at $40-800 \mathrm{MHz}$ (Mann et al. 1992), and from Ondřejov at $800 \mathrm{MHz}-2 \mathrm{GHz}$ frequency range (Jiricka et al. 1993). Some of the radio frequencies could be imaged: from the Nançay Radioheliograph (Kerdraon \& Delouis 1997) data full-disk images can be made at five frequencies: at 164, 236, 327,410 , and $436 \mathrm{MHz}$. As this event occurred in early morning hours during winter time, when solar elevation is low, we checked for atmospheric effects that could distort the observations. The emission locations on the solar disk were found to be correct until 09:00 UT, after which the ionospheric effects cannot be completely removed from the data (K.-L. Klein, private communication). Therefore we do not use positional information after 09:00 UT in this study.

\subsection{The flare and moving structures}

A GOES M 1.3 flare was observed in the NOAA AR 8858 (N25E26), starting at 08:43 UT on February 8, 2000. This active region was connected to AR 8856 (S15E15) on the southern hemisphere by transequatorial loops, but some shorter loops were also visible in between the two active regions. Figure 1 presents the evolution of the regions in soft X-rays during February 5-8.

The first flare brightening was seen in the TRACE $171 \AA$ EUV image taken at 08:43:20 UT, although some structural changes were already visible in the previous image at 08:42:35 UT. The single flaring loop soon expanded into a 

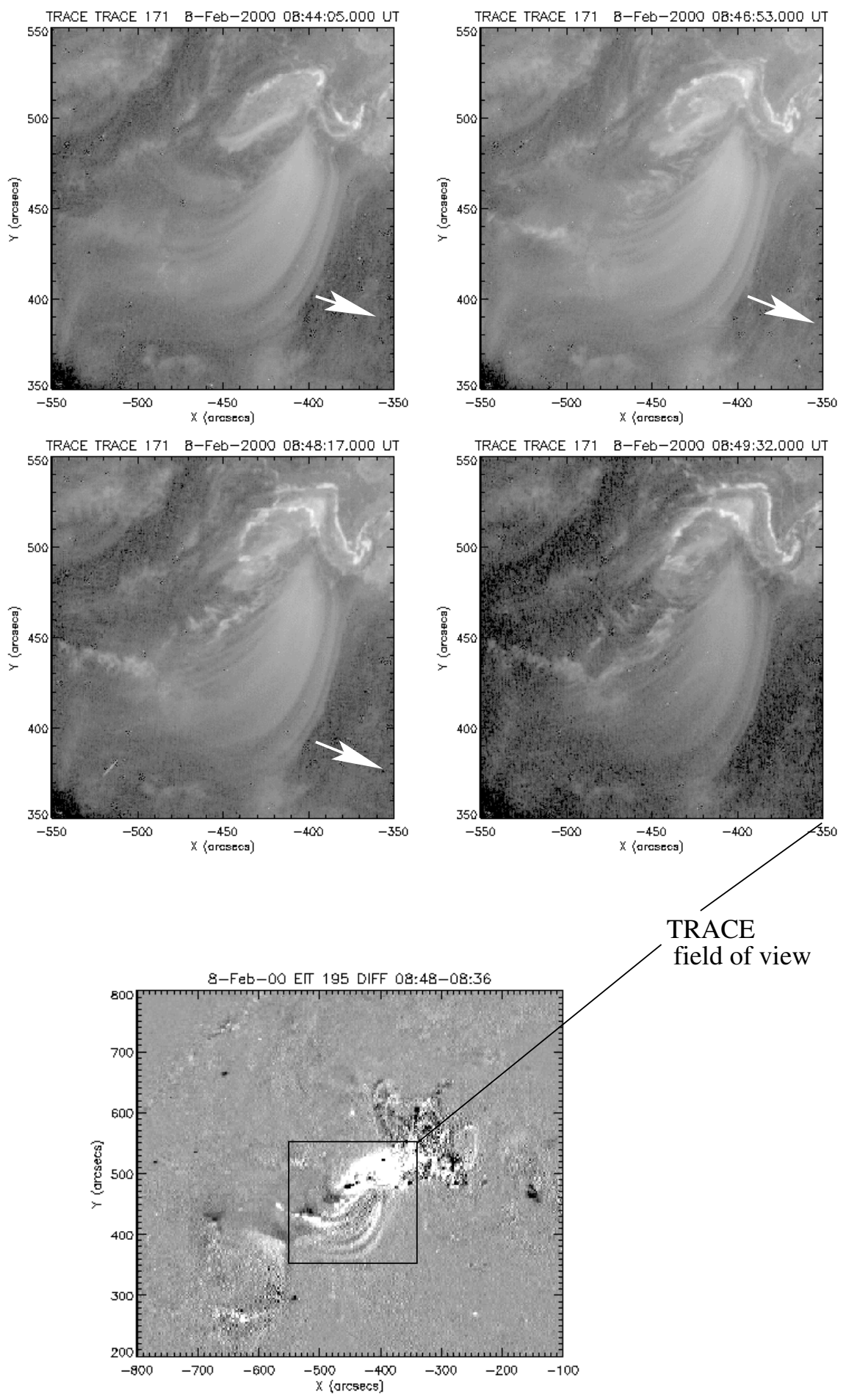

Fig. 2. EUV images of the flare region: at the top: TRACE 171 A images at 8:44:05, 8:46:53, 8:48:17, and 8:49:32 UT showing the westward moving fan-like structure (direction of movement shown with the white arrow). The speed of the moving fan was estimated to be around $100 \mathrm{~km} \mathrm{~s}^{-1}$. The two separating flare ribbons are also visible. At the bottom: SOHO EIT difference image at 08:48-08:36 UT. EIT dimming regions correspond well to the decreased brightness areas in the TRACE images - i.e. areas uncovered by the moving fan. The TRACE field view is indicated by the box in the EIT difference image.

two-ribbon flare, see Fig. 2. A fan-like structure was observed in EUV, attached to the flaring region. The fan was moving westward at a speed of about $100 \mathrm{~km} \mathrm{~s}^{-1}$ (direction indicated by the white arrows in Figs. 2 and 4). This movement is also obvious in the SOHO EIT difference images, as shown in the panel at the bottom of Fig. 2, which also shows the TRACE field of view.

In soft X-rays (Yohkoh SXT) the flare was first seen as a bright loop, with hard X-ray emission (Yohkoh HXT) coming from the loop footpoints. SOHO MDI magnetograms show 
MDI 08:03 UT ROTATED HXT LO 14-23 keV 08:48:19 UT

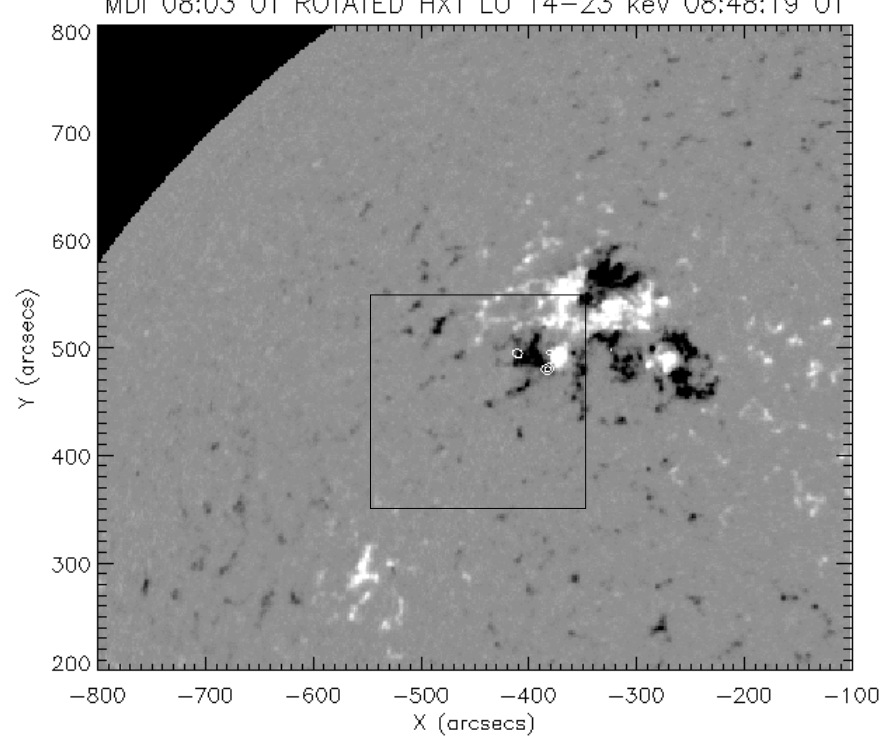

Fig. 3. SOHO MDI magnetogram from 08:03 UT shows the pre-flare multipolar active region. Yohkoh hard X-ray sources at 08:48:19 UT (HXT LO channel, 14-23 keV) are plotted over in white contours. The flare starts over a compact bipolar region, and hard X-rays are observed near the footpoints of a small X-ray loop. The TRACE field of view is indicated by the box.

that the footpoint positions were near the positive and negative fields of a compact bipolar region that was located at the edges of a large complex multipolar active region, see Fig. 3. This multipolar region was also connected to the smaller positive polarity region near $X=-550, Y=300$ (arcsec from the disk center) and to the multipolar active region near $X=-400$, $Y=-100$ in the sourthern hemisphere.

Several moving soft X-ray structures were observed to originate from the flaring region or regions nearby. These structures had the shape of expanding loops, except one that could also be interpreted as a wave front (with spherical form and high speed). The moving soft X-ray structures are summarised in Fig. 4. The projected speeds of these structures vary between $120 \mathrm{~km} \mathrm{~s}^{-1}$ and $720 \mathrm{~km} \mathrm{~s}^{-1}$. The fastest of them was a "bubble"like front moving eastwards (black solid line, expanding to the left in Fig. 4).

The EUV and soft X-ray (SXR) moving structures can be listed in order of appearance (letters indicating the features shown in Fig. 4):

a: moving fan westwards, from 08:44 UT onwards, projected speed around $100 \mathrm{~km} \mathrm{~s}^{-1}$ (EUV);

b: loop ejection towards the southeast, 08:48:50-08:49:22 UT, projected speed between 220 and $520 \mathrm{~km} \mathrm{~s}^{-1}$ (SXR);

c: expanding structure towards the northeast, 08:48:56-08:49:28 UT, projected speed between 320 and $540 \mathrm{~km} \mathrm{~s}^{-1}$ (SXR);

d: expanding "bubble" eastwards, 08:50:28-08:51:28 UT, projected speed around $720 \mathrm{~km} \mathrm{~s}^{-1}$ (SXR);

e: slow front northwards, projected speed around $120 \mathrm{~km} \mathrm{~s}^{-1}$ (SXR).
The moving structures $a, b$, and e were located close to the flaring region, and deduced from the ejection trajectories the structures $\mathrm{c}$ and $\mathrm{d}$ appeared to originate from the vicinity of a $Y$-shaped structure, at $X=-550, Y=380$ arcsec from the disk center (Fig. 2 bottom image, Fig. 4). The ejected SXR b-loop was located on the "inside" of the moving EUV fan, but the SXR ejection was observed several minutes after the EUV fan started moving. The spherical SXR "bubble" is shown in detail in Fig. 4.

\subsection{EUV and soft $X$-ray dimmings}

The first signs of dimming were seen in the EIT difference image at 08:48-08:36 UT, Fig. 2 (bottom image). The first dimmings appeared at the footpoints of loops connecting to the active region but also at remote sites, near the $Y$-shaped structure and also at $X=-670, Y=430$. This first dimming is also visible in the TRACE images, although because of the smaller field of view only part of it can be seen. The bright fan-like structure shifts westwards with a speed of about $100 \mathrm{~km} \mathrm{~s}^{-1}$, and the area uncovered by the fan is less bright. The EIT dimming region and the earlier position of the fan are in agreement (i.e. this part of the dimming is due to the fan movement). The moving EUV fan consists of a loop-like structure near the flare ribbons, and a fuzzy moving structure at the other end, but the fan does not appear to connect to the transequatorial loops.

The EUV dimmings then spread out to a larger area, shown in the difference images at 09:00-08:48 UT and 10:00-08:36 UT in Fig. 5. Part of this dimming spreads from the active region towards the East and is bordered by the smaller active region in the south-east and the active region belt near the equator. The second part of the dimming region consists of two loop-like structures that elongate to the southern hemisphere. In the first EUV difference image only the footpoints of these two loop-like structures are dimmed (seen as dark areas near the active region in the southern hemisphere, the deepest one of them indicated by a white arrow in Fig. 5a). In the second EUV difference image the dimmings have deepened into two loop-like structures (pointed by the white arrows in Fig. 5b).

A loop-like dimming was also observed in soft X-rays (Fig. 5c). The last full-disk long exposure Yohkoh SXT image before partial frame flare mode was taken at 08:33 UT, and the next after the flare at 10:00 UT. In the SXT difference image at 10:00-8:33 UT we see one wide loop-like structure that lies approximately in between the two loop-like EUV dimmings. A faint SXT dimming is however located over the right-hand side loop-like EUV dimming (indicated by the black arrow on the right in Fig. 5c). Figure 5d shows the SXT dimming plotted in contours over the EUV dimming. If we take into account that the loops seen in EUV and soft X-rays represent plasma at different temperatures and at different heights, and also take into account possible projection effects, we can make the conclusion that the dimmed loops seen in EUV and soft X-rays are partly separate, but have connectivities to the same active regions. 


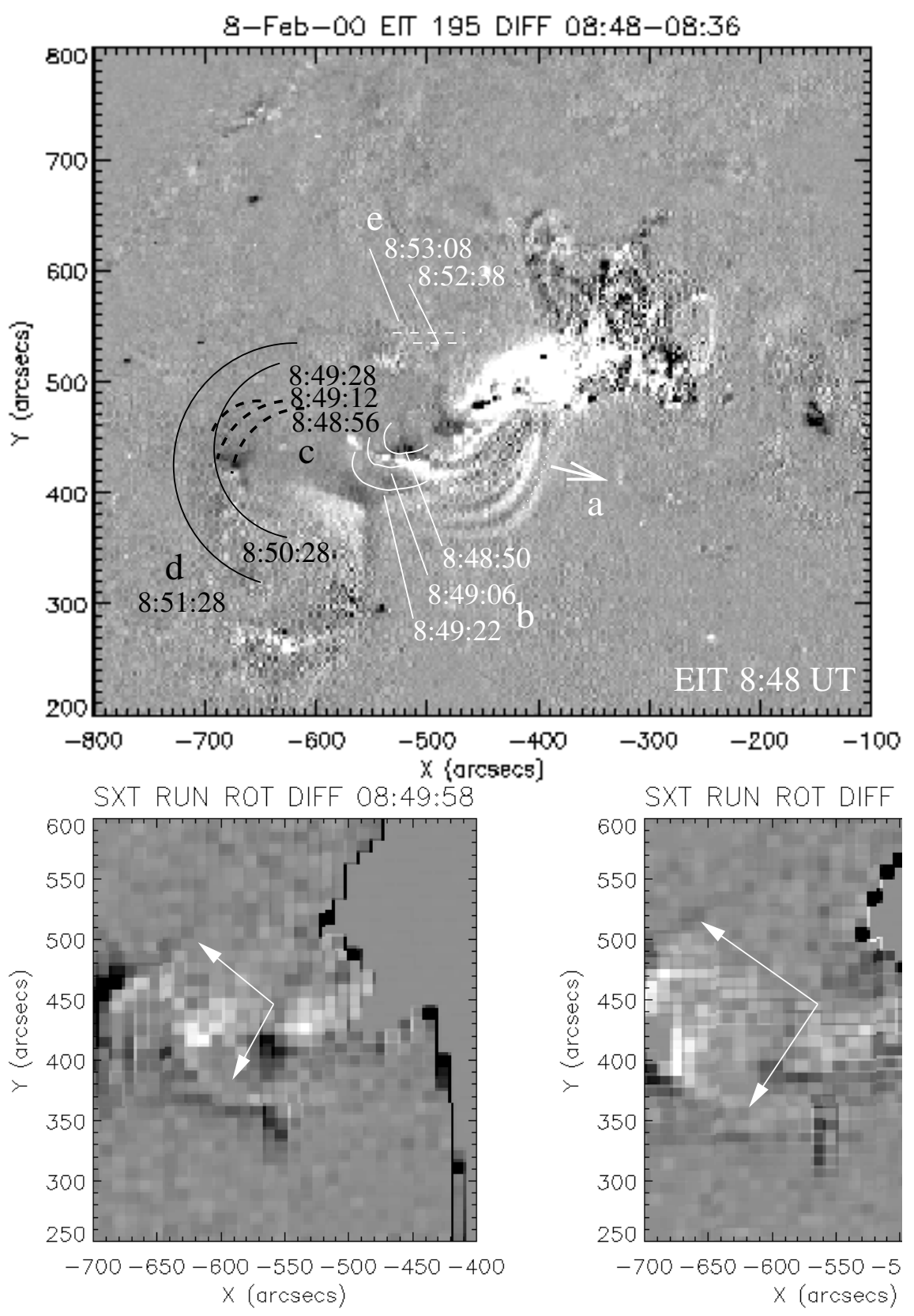

Fig. 4. At the top: times and positions of the EUV and soft X-ray (SXR) loops and waves, superposed on the EIT difference image at 08:48-08:36 UT. White arrow shows the direction of the EUV moving fan-like structure a), with estimated speed of $100 \mathrm{~km} \mathrm{~s}^{-1}$. The first ejected SXR loop b), shown at 08:48:50 UT-08:49:22 UT (white lines) had an estimated projected speed between 220 and $520 \mathrm{~km} \mathrm{~s}^{-1}$. The SXR expanding structure c), shown at 08:48:56-08:49:28 UT (black dashed lines) was estimated to have projected speed between 320 km $\mathrm{s}^{-1}$ and $540 \mathrm{~km} \mathrm{~s}^{-1}$. The front of the expanding SXR "bubble" d), shown at 08:50:28 and 08:51:28 UT (black lines) had a projected speed around $720 \mathrm{~km} \mathrm{~s}^{-1}$. The northwards moving SXR structure e), shown at 08:52:38 and 08:53:08 UT (white dashed lines) was estimated to have a projected speed around $120 \mathrm{~km} \mathrm{~s}^{-1}$. At the bottom: the expanding SXR "bubble" is shown at two selected time intervals, in rotated running difference images (Yohkoh SXT). Arrows indicate the expanding structures. The (projected) speeds were determined from positional changes (arcseconds on the disk) of the loop fronts over radials in the direction of the loop movements (different for each). No corrections were made for heights or changing line of sight.

\subsection{Fast drift and narrow-band radio bursts}

The complete radio event is shown in the dynamic spectra in Fig. 6. The event began with two separate reverse slope (RS) type III bursts at 08:44:20 UT and 8:46:05 UT ("A" and "B" in Fig. 6). The bursts were visible only at a narrow band between 320 and $500 \mathrm{MHz}$.
During 8:48:30-8:50:30 UT a new group of type III bursts occurred. Some of them were J-bursts (incomplete U-bursts), within the group marked "C" in Fig. 6. The bursts in the $\mathrm{C}$ group had a turn-over frequency around $150 \mathrm{MHz}$. The turnover frequency was observed to change in time towards the lower frequencies. Some of the descending drifts in these bursts (drifts towards the higher frequencies, i.e. beams going down 

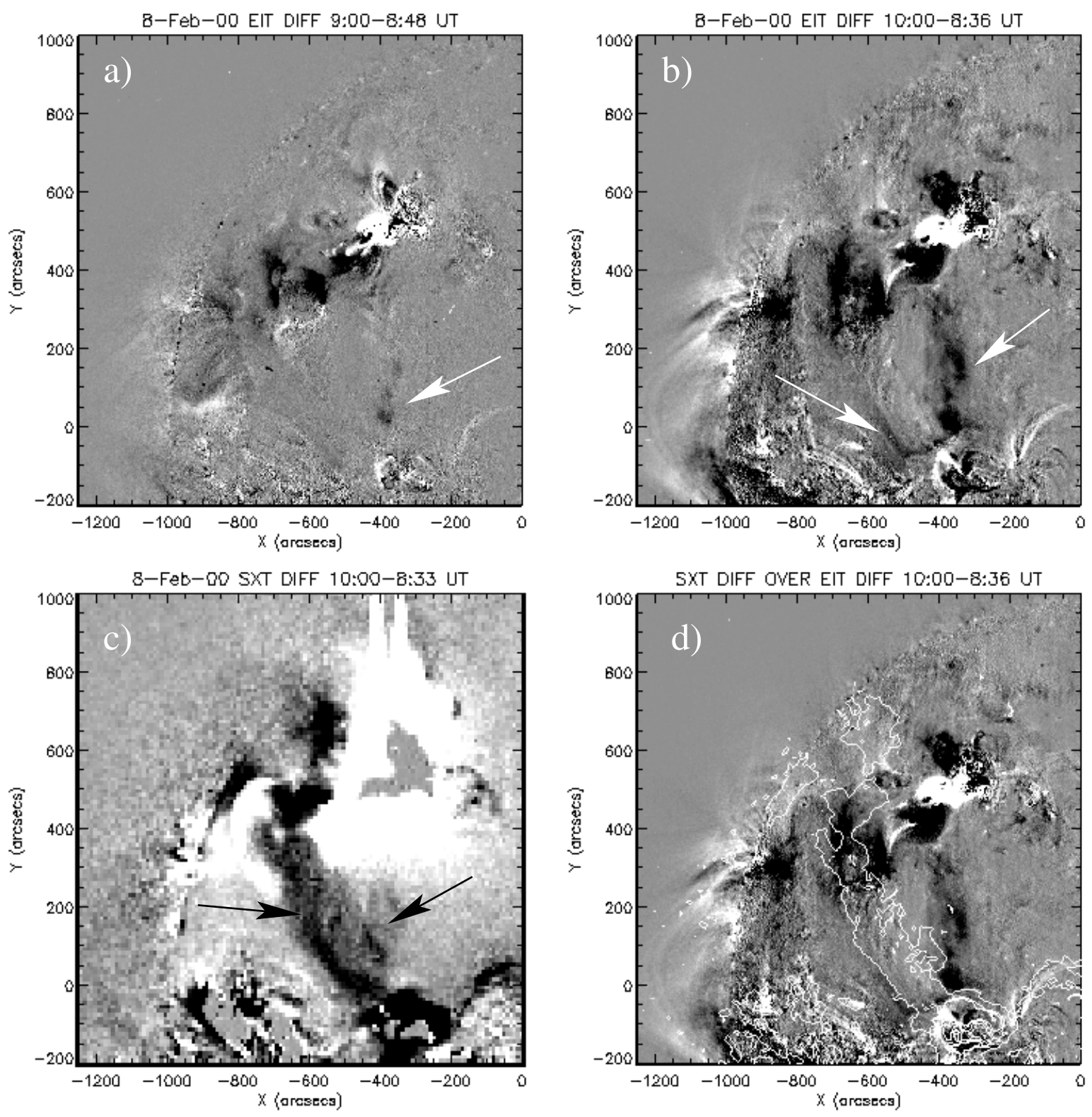

Fig. 5. EIT difference images at 09:00-08:48 UT a) and at 10:00-08:36 UT b), Yohkoh SXT difference image at 10:00-08:33 UT c), and EIT difference image at 10:00-08:36 UT with overplotted contours of the SXT difference image from the same time d). The disappeared SXT and EIT transequatorial loops are indicated by arrows. It is evident that the footpoints of the dimmed loops connect to the same active regions.

in the solar atmosphere) are easy to follow in the spectra, but in many cases the ascending and descending branches get mixed at higher frequencies. The J-burst group was followed by another group of narrow-band fluctuations at 8:51:30-8:52:30 UT (marked "D" in Fig. 6). The C and D burst groups are shown in more detail in the dynamic spectra in Fig. 7.

In hard X-rays, the $14-23 \mathrm{keV}$ count rate started to rise at $8: 43: 15$ UT, although the main enhancement did not begin until 8:47:30 UT, see Fig.6. The higher energy channels did not start to observe until 08:48 UT. They show basicly the same evolution. The hard X-ray emission peak was reached at 8:48:25 UT, well before the main activity phase in the decimetric-metric radio band.

The radio emission locations could be imaged at five frequencies simultaneously, using the Nançay Radioheliograph recordings at $164,236,327,415$, and $432 \mathrm{MHz}$. The location of the first "A" and "B" RS-bursts, at $327 \mathrm{MHz}$, are shown in Fig. 8a, marked with a black circle. Due to the narrow band we cannot image these bursts at several frequencies (i.e. at many different heights) but we can say that the bursts were drifting towards the higher frequencies. Higher frequency basicly means plasma emission at higher density - i.e. the electron beams were moving down in the solar atmosphere at that location. Plasma emission at this frequency range indicates burst exciter heights of at least $100000 \mathrm{~km}(0.15 \mathrm{R})$ above the photosphere. Projection effects are therefore non-negligible, and in this case the RS burst sources could have been located over the flaring region.

The emission locations of one of the most prominent J-bursts in the "C" group are shown in Fig. 8b. Black triangles 


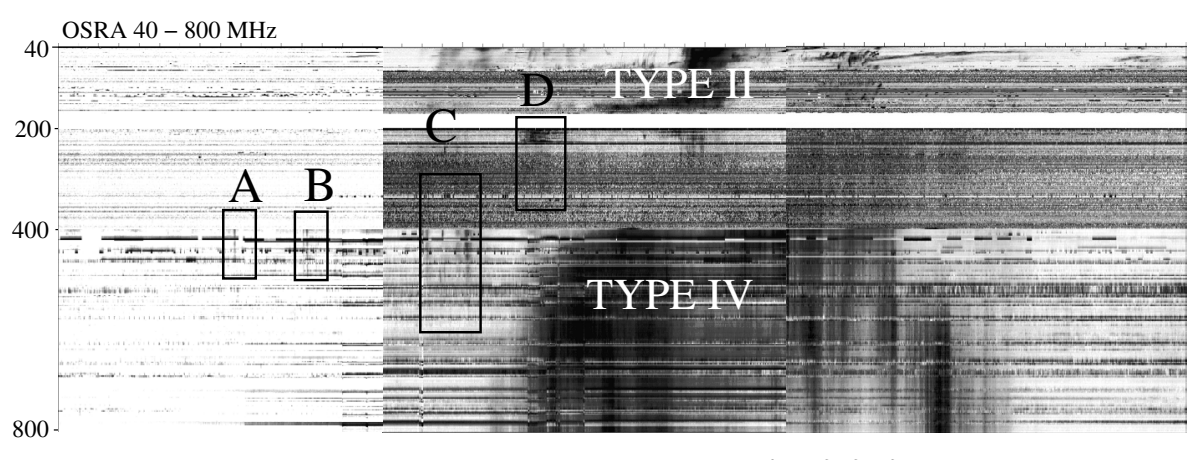

$\mathrm{H} \times \mathrm{T} 14-23 \mathrm{keV}$ (cts $/ \mathrm{s} / \mathrm{SC}$ )

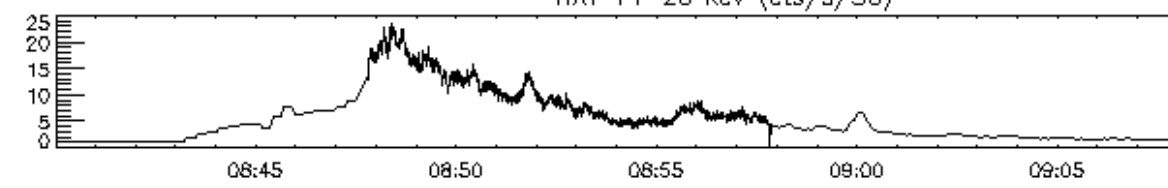

$\mathrm{HXT} 23-33 \mathrm{keV}(\mathrm{cts} / \mathrm{s} / \mathrm{SC})$

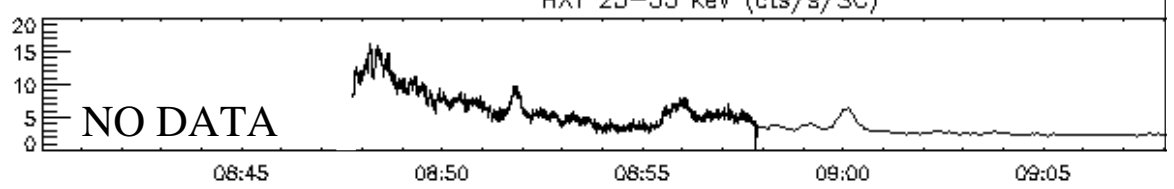

GOES 8 X-Rays:

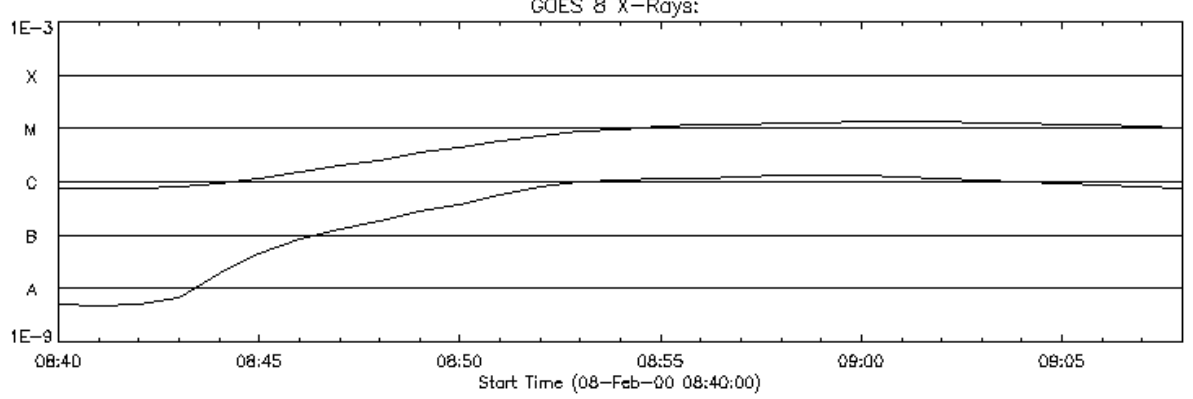

Fig. 6. At the top: radio spectra at the $40-800 \mathrm{MHz}$ frequency range from OSRA/Tremsdorf at 08:40-09:08 UT. The different spectral parts in the composite image have been enhanced to show less intense emission in more detail. The radio type III bursts are marked in the spectra with boxes A-C: A and B are single reverse slope (RS) bursts around 08:44 and 08:46 UT. C is a group of J-type bursts around 08:49-08:50 UT. D is a group of narrow-band fluctuations around 08:52 UT. Radio type II emission at the fundamental (near $80 \mathrm{MHz}$ ) and second harmonic (near $160 \mathrm{MHz}$ ) are visible during 08:53-08:56 UT. Radio type IV continuum emission starts around $800 \mathrm{MHz}$ at 08:51 UT, and the burst envelope drifts towards the lower frequecies. Some parts of the radio spectra are shown in detail in Fig. 7. In the middle: Yohkoh HXT flux curves in the $14-23 \mathrm{keV}$ and $23-33 \mathrm{keV}$ energy channels. At the bottom: GOES soft X-ray flux at $12.5-1.5 \mathrm{keV}$ and at $25-3 \mathrm{keV}$.

indicate the positions at each time and frequency, and the white arrows give the direction of the burst. The times and frequencies were marked in the dynamic spectra in Fig. 7 with black triangles. The burst traces a large loop-like structure towards the South, and towards the AR 8856. The resolved RS branches in the J-burst group all trace the same path. The EIT difference image over which the radio sources are plotted in Fig. 8 indicates that the J-burst path lies over the right-hand side loop-like EUV dimming, which was shown in detail in Fig. 5.

The emission locations of the narrow-band fluctuations in the "D" group spread wider. In Fig. 8c the burst locations at 8:52:04 UT at $236 \mathrm{MHz}$ are plotted, i.e., we have three different emission locations simultaneously at one frequency. The spread of emission locations at one single frequency indicates that electrons now have access from the flaring region to many more separate loop systems than before. The last emission features near the transequatorial loop along which the J-bursts in the "C"'-group were located were visible at 8:52:30 UT at 410 and $432 \mathrm{MHz}$.

\subsection{Radio type II burst and decimetric continuum}

The dynamic radio spectra at decimetric-metric wavelengths shows a radio type II burst at the fundamental (near $80 \mathrm{MHz}$ ) and second harmonic (near $160 \mathrm{MHz}$ ) starting at 08:53 UT. Both lanes are shown in Fig. 6 and the second harmonic lane in detail in Fig. 7. The start of the 2nd harmonic of the type II burst is not clear, as the start is mixed with the narrow-band fluctuations.

The type II burst could be imaged at $164 \mathrm{MHz}$, and the burst locations at 8:53, 8:54, and 8:55 UT are shown in Fig. 8d (white boxes). The times were indicated in the dynamic spectra in Fig. 7 (white boxes inside the type II lane). The burst locations during 08:53-08:54 UT show a trajectory towards the South 

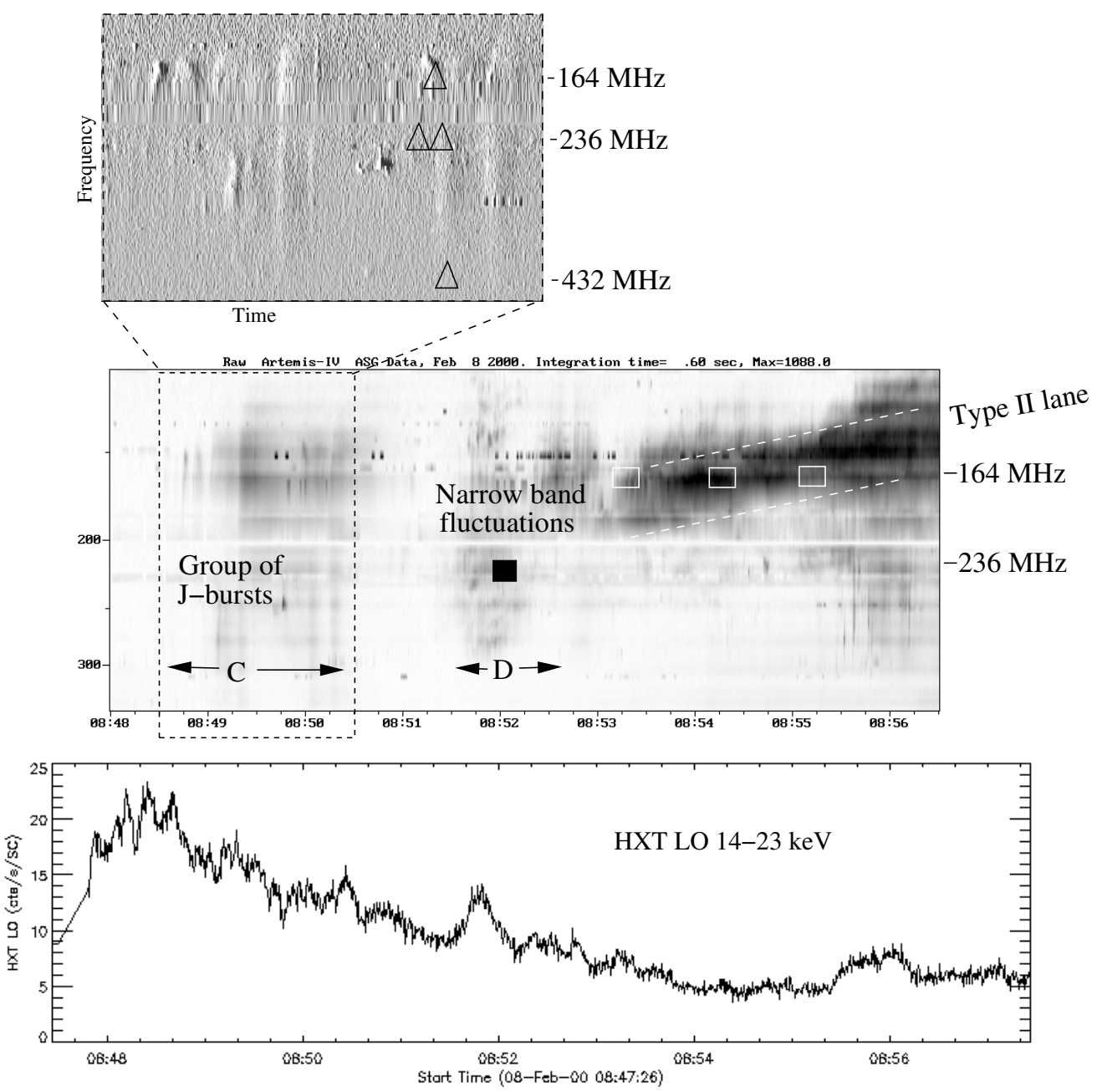

Fig. 7. Artermis-IV dynamic spectra at 100-350 MHz during 08:48-08:56:30 UT, and a detail of the time derivative of the Artermis-IV intensity during the J-burst group at 08:48:30-08:50:30 UT. The times of the J-burst group (C), the narrow-band fluctuations (D), and the type II burst (at second harmonic) are marked on the plot. The black triangles indicate the times and frequencies of the locations of one of the J-burst branches, shown in Fig. 8b. The black box gives the time of one narrow band burst at $236 \mathrm{MHz}$, the multiple locations of which are shown in Fig. 8c. The white boxes in the spectra indicate the times of the type II burst locations shown in Fig. 8d, at 164 MHz. At the bottom Yohkoh HXT LO channel counts are shown for comparison.

and towards the inner parts of the disk, but around 08:54 UT the burst location shifts more towards the limb. It can be noted that this shift happens above the equatorial active region belt, above which a coronal streamer is formed.

The decimetric-metric continuum, marked "type IV" in Fig. 6, appears in the dynamic spectra around 8:50:40 UT at $800 \mathrm{MHz}$. It is visible also in the $0.8-2.0 \mathrm{GHz}$ range (Ondřejov dynamic spectra). The envelope of the type IV burst drifts towards the lower frequencies, and it reaches $400 \mathrm{MHz}$ around 8:53:00 UT. The emission location of the type IV burst at 410 and $432 \mathrm{MHz}$, where it cannot be confused with other spectral features, is near the region where the first RS type III bursts ("A" and "B") occurred. The position of the type IV emission location is marked in Fig. 8d (black circle). When compared with the SXT difference image in Fig. 5c, the type IV emission location is observed to be over a strong SXT dimming region. The type IV radio emission source is almost point-like, with no elongated structures. The emission source centroid does not move much. During the type IV burst the total positional shift is less than 50 arcsec, indicating that this is an almost stationary type IV burst. The metric continuum emission ends around 09:04 UT.

\subsection{Halo coronal mass ejection}

A halo CME was first seen in the LASCO C2 image at 09:30 UT (LASCO CME Catalogue ${ }^{1}$, Catholic University of America). The estimated CME speed was around 1200-1300 $\mathrm{km} \mathrm{s}^{-1}$ at 9:30-10:00 UT, slowing down to $900 \mathrm{~km} \mathrm{~s}^{-1}$ at 12:42 UT. The most compact CME structure was seen over the North-East limb. This event is one of the flare-fast CME events listed by Zhang \& Golub (2003). The LASCO C2 image at 09:30 UT is shown in Fig. 9. Arrows point to the CME front over the North-East limb.

The LASCO CME Catalogue also gives linear and 2nd order fits to the CME positions at different times. A linear fit to

\footnotetext{
${ }^{1}$ http://cdaw.gsfc.nasa.gov/
} 

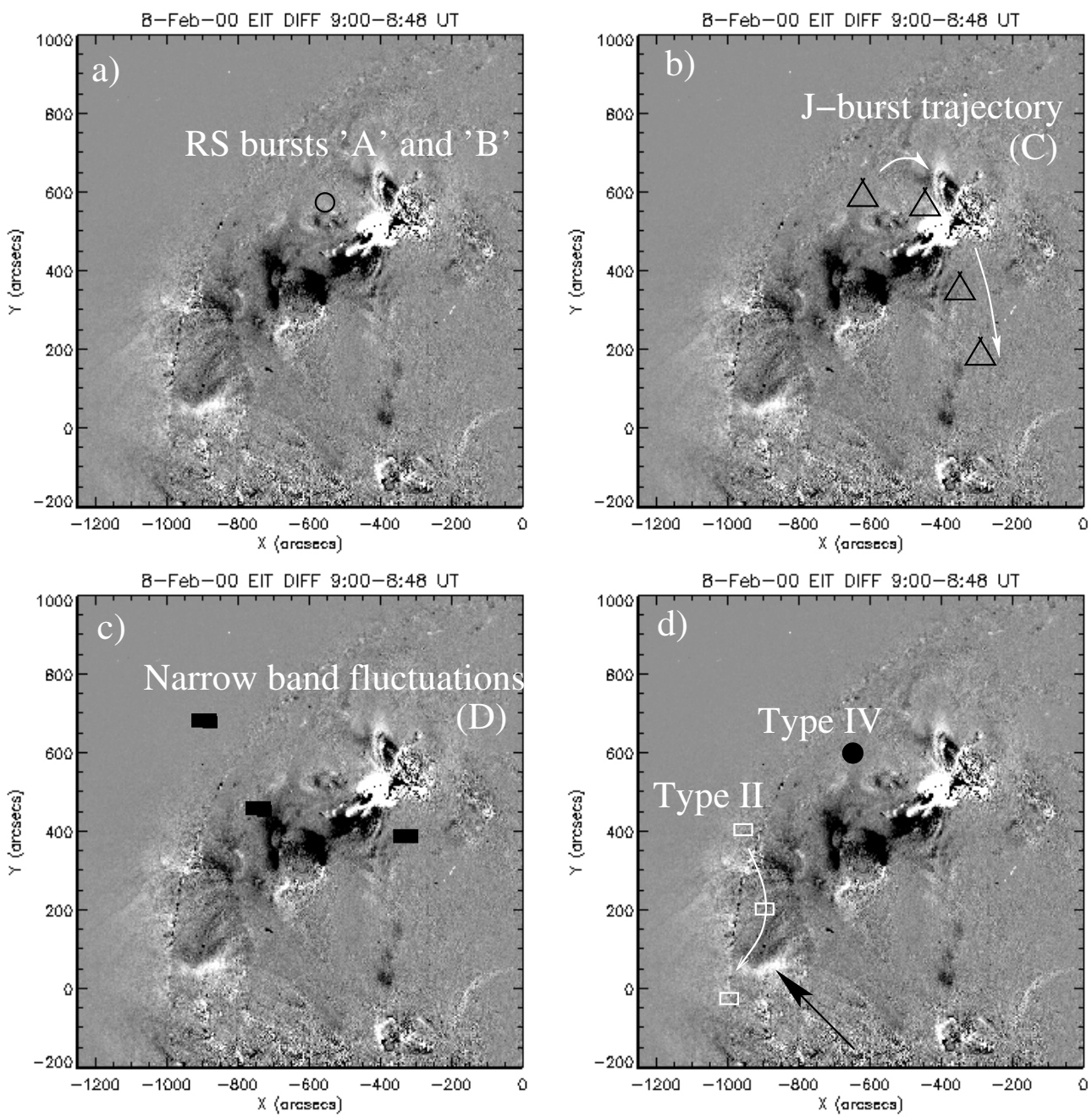

Fig. 8. a): The location of the first reverse slope (RS) bursts "A" and "B" at 8:44:20 and 8:46:05 UT (O). b): One of the radio type III J-bursts in the "C" group, the emission locations are marked at 8:49:55 UT at $236 \mathrm{MHz}, 8: 50: 02 \mathrm{UT}$ at 164 MHz, at 8:50:04 UT at 236 MHz, and at 8:50:06 UT at $432 \mathrm{MHz}(\Delta)$, arrow shows the temporal evolution. The J-burst becomes visible first at $236 \mathrm{MHz}$ and drifts towards the lower frequencies - the spectral turnover occurs around $140 \mathrm{MHz}$ - and after that the burst drifts towards the higher frequencies, i.e. it goes down in the solar atmosphere. The frequencies and times were indicated with $\Delta$ in the dynamic spectra in Fig. 7. c): The three separate locations of the narrow-band burst at 8:52:04 UT at $236 \mathrm{MHz}(\mathbf{m})$ belonging to the burst group "D". d): Type II burst locations at 164 MHz at 08:53, 08:54, and 08:55 UT (口). Arrow shows the spatial movement in time, i.e. it first moves down and towards the inner part of the disk but then shifts slightly back towards the disk edge. The black arrow points to the bright region where the type II exiter is thought to collide with the active region belt/coronal streamer. The location of the stationary metric type IV emission is also indicated $(\bullet)$. All the radio sources are plotted over the EIT difference image at 9:00-8:48 UT. Dimming regions (depleted matter) are shown as dark regions, while bright regions represent more hot or dense matter.

all the observations would place the CME front at $R=0.8 R_{\text {sun }}$ (i.e. near the flaring region) around 09:00 UT, and a 2nd order fit around 09:10 UT. However, the first three LASCO observations can be fitted with another height-time trajectory, and a linear fit to the three positions give a CME location at $R=0.8 R_{\text {sun }}$ around 08:45 UT. The February 8, 2000 event is also among the CME-type II events listed by Leblanc et al. (2001). They have used a second order polynomial fit and extrapolated the measurements to the location of the active region, also correcting for the position angle and radial speed, and give 08:47 UT as the extrapolated lift-off time at the active region (with $5 \mathrm{~min}$ uncertainty). The CME start location near the AR with a speed of $1300 \mathrm{~km} \mathrm{~s}^{-1}$ would place the CME front near the solar limb around 08:49...08:51 UT.
In comparison, the first $\mathrm{RS}$ radio burst was observed at 08:44:20 UT, the J-bursts along the transequatorial loop appeared between 08:48:30 and 08:50:30 UT, the start of the type IV burst at 08:50:40 UT, and the start of the type II near the limb at 08:53 UT. The radio source locations are plotted in summary in Fig. 9, and a summary of observed events is presented in Table 1.

\section{Interpretation of observations}

\subsection{Early disk signatures of the development of the halo CME}

The flare started almost simultaneously in EUV and soft and hard X-rays. Several loop ejections and one possible wave front 


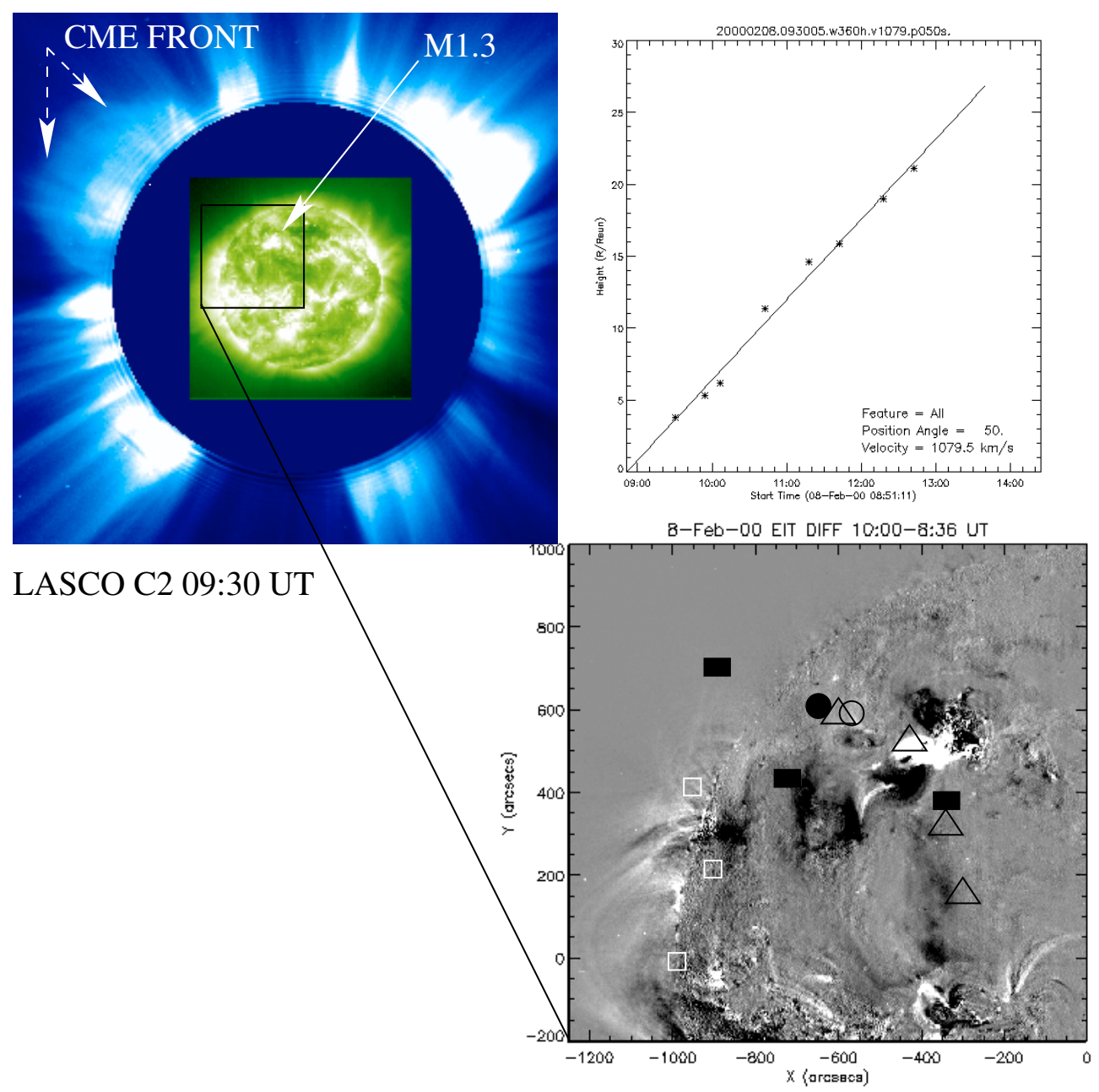

Fig. 9. The start of the halo CME was observed by SOHO LASCO C2 at 09:30 UT over the North-East limb, and the location of the M 1.3 GOES flare in AR 8858 is also indicated in the image. The height-time plot from the LASCO CME Catalog is also shown. The estimated onset time of the CME depends on the fit and the number of fitted data points. The different types of radio burst sources are marked over an EIT difference image at 10:00-08:36 UT, which shows the deepest EUV dimmed regions on the solar disk: $\bigcirc$ RS bursts "A" and "B"; $\Delta$ J-burst belonging to group "C"; — Narrow-band burst belonging to group "D"; $\square$ Type II burst; • Stationary metric type IV burst.

were observed in soft X-rays, propagating in different directions. Small dimming regions were first observed at loop footpoints, also at remote sites some distance away from the flare core region. It should be remembered that dimmings do not always indicate depleted matter (i.e. a plasma flow after opening of field lines), as dimmings can be produced by a decrease in electron density or by a change in temperature. Structures that become elongated and thus less dense can be observed as dimming regions in EUV images. Dimmings can also happen because material either cools or heats until it no longer emits in the observed passband (Harra \& Sterling 2001). In our event the first dimmed regions were related to the moving EUV fan, and the dimmings were caused by the underlying cooler chromospheric material being exposed. These first small dimmed regions do not seem to be directly related to the more prominent large-scale dimmings that were caused by depleted matter, associated with the CME itself.

The radio J-bursts occurred near the time of the first soft $\mathrm{X}$-ray loop ejections. The turn-over frequencies of the J-bursts drifted towards the lower frequencies, indicating that either the electron beams had access to higher loop structures or that the loops themselves were rising. There was no significant hard $\mathrm{X}$-ray emission connected with the "C" group of U and J-bursts (Fig. 7). The group of narrow-band fluctuations ("D") and the type III burst group below the type II lane were, however, associated with definite rises in the hard X-ray count rates. This and the fact that the starts and ends of the J-bursts were mixed, could be signs of magnetic confinement (mirroring): electrons trapped in the large loops were not able to escape. This type of N-bursts (type $\mathrm{U}$ with a third, ascending branch) have been reported earlier by Caroubalos et al. (1987).

The radio J-bursts traced a large loop-like structure towards the southern hemisphere. This radio emission path was located over an intense loop-like EUV dimming that was observed after the radio bursts. Soft X-ray images from earlier dates confirm that transequatorial loops and possibly coronal X-points were present and connected the two active regions on the different hemispheres. The large loop-like EUV and soft X-ray dimmings indicate that at least part of the CME was transequatorial loop material. 
Table 1. Summary of observed events.

\begin{tabular}{|c|c|c|}
\hline Time (UT) & Event & Comments \\
\hline $08: 42: 35$ & Flare brightening in EUV & TRACE, NOAA 8858 at N25E26 \\
\hline $08: 42: 40$ & $\mathrm{X}$-ray flare onset & GOES, Yohkoh HXT \\
\hline $08: 44$ & Start of moving EUV "fan" & $100 \mathrm{~km} \mathrm{~s}^{-1}$, towards West (TRACE, EIT) \\
\hline 08:44:20 & RS type III burst & NE of AR (radio) \\
\hline 08:46:05 & RS type III burst & NE of AR (radio) \\
\hline $08: 47: 40$ & Hard X-ray rise & impulsive burst start in the $14-53 \mathrm{keV}$ range \\
\hline 08:48 & First signs of EUV dimming & EIT difference image at 08:48:12-08:36 UT \\
\hline $08: 48: 24$ & Hard X-ray maximum & $\begin{array}{l}\text { several peaks in } 14-53 \mathrm{keV} \text { (Yohkoh HXT), location over flare ribbons } \\
\text { (two-ribbon flare) }\end{array}$ \\
\hline 08:48:30 & Start of J-bursts & along transequatorial loop, towards South from AR (radio) \\
\hline$-08: 48: 50-$ & Loop ejection & $220-520 \mathrm{~km} \mathrm{~s}^{-1}$, towards SE (Yohkoh SXT) \\
\hline$-08: 48: 56-$ & Expanding structure & $320-540 \mathrm{~km} \mathrm{~s}^{-1}$, towards NE (Yohkoh SXT) \\
\hline$-08: 50: 28-$ & Expanding "bubble" & $720 \mathrm{~km} \mathrm{~s}^{-1}$, towards East (Yohkoh SXT) \\
\hline 08:50:30 & End of J-bursts & \\
\hline 08:50:40 & Start of type IV & decimetric continuum begins at $800 \mathrm{MHz}$ (radio) \\
\hline 08:51:30 & Start of narrow-band bursts & widespread locations (radio) \\
\hline 08:52:30 & End of narrow-band bursts & \\
\hline$-08: 52: 38-$ & Moving front & $120 \mathrm{~km} \mathrm{~s}^{-1}$, towards North (Yohkoh SXT) \\
\hline 08:53 & Start of type II & $\begin{array}{l}\text { 2nd harmonic at } 160 \mathrm{MHz} \text {, near NE limb moving towards South, } \\
\text { fundamental at } 80 \mathrm{MHz} \text { (not imaged), several lanes after 08:58 UT (radio) } \\
\text { velocity estimate } 806 \mathrm{~km} \mathrm{~s}^{-1} \text { (Caroubalos et al.) }\end{array}$ \\
\hline 09:00:00 & Soft X-ray flare maximum & GOES M 1.3 \\
\hline 09:04 & End of type IV & stationary, location NE of AR (radio) \\
\hline 09:30:05 & First appearance of CME in LASCO C2 & $\begin{array}{l}\text { compact structure at } 3.81 R_{\text {sun }} \text { in NE } \\
\text { estimated speed } 1300 \mathrm{~km} \mathrm{~s}^{-1} \text { (LASCO CME Catalog) }\end{array}$ \\
\hline
\end{tabular}

Type II radio bursts are known to be formed by shocks, either by piston-driven shock fronts or by (flare) blast waves. The emission itself is plasma emission at the fundamental or second harmonic plasma frequency, which depends on the local plasma density. Caroubalos et al. (2004) have estimated the velocity of the type II burst driver on February 8, 2000 to be about $806 \mathrm{~km} \mathrm{~s}^{-1}$, using the Newkirk density model and the burst frequency drift. One of the moving soft X-ray features, the expanding bubble-like front (the front locations at two different times were outlined in Fig. 4), had relatively high projected speed around $720 \mathrm{~km} \mathrm{~s}^{-1}$ and a spherical shape. The place where the soft X-ray bubble first became visible was near the "Y" structure, away from the flare site. Remote sites of shock formation have been reported earlier by Hudson et al. (2003). The soft X-ray bubble is the best shock wave candidate that could produce a shock front capable of driving a type II burst. However, the extrapolated locations of the "bubble" do not reach the type II burst locations in time to definitely identify it as the burst driver. On the other hand, the shock front could accelerate and one cannot rule out projection effects at high latitides and near the solar limb.

Because of the uncertainty in the CME start (time and location) we cannot exclude the possibility that the soft X-ray bubble-like front was associated with the CME, although the direction of the bubble movement does not fit the direction of movement of the CME front. The large-scale EUV dimmings that appeared between 08:48 and 09:00 UT also make it possible that depleted matter was acting as a piston and created the type II emission. Due to the low EIT image cadence and the Yohkoh SXT partial frame observing mode, no moving coronal matter could be imaged. The shift in the type II burst position after 08:55 UT can be explained by the burst exiter meeting the active region belt/coronal streamer. An off-limb brightening in EUV has been reported by Glover et al. (2003), when a coronal streamer was displaced by an eruption in connection with a disappearing transequatorial loop.

Pohjolainen et al. (2001) observed strong decimetric type IV continuum emission over an EUV dimming region during the May 2, 1998 event, and interpreted it as accelerated electrons in expanding large-scale loops associated with the CME. In the February 8, 2000 event the type IV continuum was drifting in frequency but not moving much spatially. This could be a projection effect again, if the burst source was moving towards the observer. However, decimetric type IV emission is still poorly understood, and the complicated interpretation of the structures has been based on loss-cone emission processes (Benz 2003).

\subsection{Comparison to the large scale changes observed on May 2, 1998}

Similarities can be found between the flare/halo CME event of February 8, 2000 and the one on May 2, 1998. Both of these events were associated with a two-ribbon flare, EUV dimmings, wave-like phenomena, disappearing transequatorial loops, electron beams travelling along the large loops (J-bursts in radio), and decimetric type IV emission in the late phase of the flare. We now list and discuss the main similarities between 
the two events:

- The radio emission in these events starts with J-bursts that trace electron beams travelling along large transequatorial loops. Soon after this the remote ends of these transequatorial EUV loops get dimmed. In the May 2 event the footpoint dimming near the northern AR was visible in the first EIT difference image at 13:41-13:19 UT, Fig. 6 in Pohjolainen et al. (2001), but this was not mentioned in the paper.

- Soon after the J-burst groups widely separated structures are seen at one radio frequency. In the May 2 event this is right after the start of the flash phase and before the type IIlike structures. In the February 8 event the widespread radio sources belonged to a group of narrow-band fluctuations which also occurred before the type II burst. These two radio spectral features are very similar in the dynamic spectra, the difference is that in the May 2 event the "flash" went up to high frequencies probably masking the less intense narrow-band fluctuations.

- In the later phase large-scale, loop-like EUV and soft X-ray dimmings are formed in both events. The X-ray and EUV dimming loops are not completely cospatial, but lie close to each other. Wang et al. (2002) extrapolated the magnetic fields of the May 2, 1998 event and their extrapolations show that the J-type radio bursts traced two different transequatorial loops (L1 and L2 in Fig. 6 of their paper). One loop was seen in soft X-rays and it dimmed partially, and the other loop was located over the strong EUV dimming region. Their interpretation was that the fields opened completely over the EUV dimming region and the CME material consisted mainly of depleted matter from that region, but that the field opening was only partial over the soft X-ray transequatorial loop.

- Strong type IV continuum emission was observed in both cases, starting around the same time as the wide-spread pulsations and fluctuations. Moving type IV bursts have been shown to be confined to loops and blobs of the CMEs, the emission mechanism being either gyrosynchrotron emission or plasma emission (see review by Kahler 1992, and references therein). In May 2 the burst was a moving type IV and the emission sources were located over the largest EUV dimming region. This suggests that the May 2 decimetric moving type IV could be due to rising (depleting) EUV material with large spatial distribution, and the February 8 stationary type IV emission could be emission from trapped electrons in a much smaller volume.

- In the February 8 event the type II burst emission could be associated the moving soft X-ray "bubble". The relation of the type II burst and the CME front is unclear: if we accept the CME front locations from a fit to all the LASCO data points, the bubble and the type II burst exiter are proceeding well ahead of the CME front. If, however, we accept a fit to the first three LASCO observations the times for the bubble and the CME front at the limb come closer. On the other hand, the CME speed was measured to be around $1300 \mathrm{~km} \mathrm{~s}^{-1}$ at the beginning, the bubble speed around $720 \mathrm{~km} \mathrm{~s}^{-1}$, and the type II burst driver around $800 \mathrm{~km} \mathrm{~s}^{-1}$, making the association between the last two more probable. In the May 2 event the type II-like bursts had close association with the proceeding $\mathrm{H} \alpha$ (Moreton) wave front but they were also untypical in the way that the observed EIT-wave and the $\mathrm{H} \alpha$ Moreton wave were closely related (see discussion in Biesecker et al. 2002). Also the frequency drift rates of the type II-like bursts were higher than usual on May 2.

- The estimated CME lift-off time (defined as the time when the backwards extrapolated CME front is located at the active region) can be put near the time of the J-bursts in both cases. In the February 8 event the CME front could be located near the active region at $R=0.8 R_{\text {sun }}$ around 08:45-08:47 UT (first J-burst along transequatorial loop at 08:48 UT), and in the May 2 event at $R=0.5 R_{\text {sun }}$ around 13:35 UT (first J-burst along transequatorial loop at 13:35 UT), lift-off time taken from the 2nd order fit in the LASCO CME Catalog. In both cases the J-bursts were preceded by type III - RS type III bursts, starting 4-6 min before the J-burst emission along the transequatorial loops.

\section{Discussion}

We propose, based on the observations of the February 8, 2000, and the May 2, 1998 halo-CME events, that we see large-scale field line opening evidenced by radio emission tracing electron propagation paths along large transequatorial loops in which large scale mass depletion later takes place. Radio J-bursts trace electron beams that travel along the large transequatorial loops, just before EIT dimmings set on the footpoints of these loops. The J-bursts are therefore signatures of energetic electrons produced in rearranging magnetic fields, before the field opens up completely. The start of energy release does not seem to originate in concentrated, sheared fields linked to the active region, but originates high in the corona when the large loops first get destabilized.

The soft X-ray dimmings in the form of disappearing transequatorial loops and the EUV dimmings in similar form - but probably consisting of low-lying sets of loops - suggest that they are part of the CME material. The presence of shock waves (a clear EIT-wave-Moreton wave-type II association in the May 2 event, and a soft X-ray expanding "bubble"-type II association in the February 8 event) in both cases poses the question that shocks could initially be part of the destabilization process. The start of the type IV emission - usually interpreted as rising structures - also coincides with the end of the J-bursts, thus marking the time of field line opening.

Large scale field line opening preceding the lift-off of material is an essential part of the "breakout" model by Antiochos (1998) and Antiochos et al. (1999). They claim that the opening of fields starts at a neutral point high above the core region. The initial condition requires a multipolar complex field with coronal null points where magnetic reconnection can occur. So far only some observational evidence has been produced in support of the model (Manoharan \& Kundu 2003; Maia et al. 2003). Sterling \& Moore (2004) have suggested a mixed model with slow pre-eruption reconnection at the top of a loop system preceding a fast eruption in the sheared core region. Recently MacNeice et al. (2004) have presented the first simulations of 
the complete breakout process, showing that the strongest signatures of breakout are likely to be present during the impulsive phase of a flare, although weaker pre-flare signatures should also be present. This is in agreement with our observations, with electron beams directed downwards from the corona (RS type III bursts) before the flare impulsive phase and electron beams traveling along large transequatorial loops (J-bursts) just after the hard X-ray maximum. The magnetic field extrapolations and the pre-flare signatures of the May 2, 1998 event also suggested slow reconnection between the transequatorial loop system and the pre-existing overlying field above the sheared active region (Wang et al. 2002). In these two events the large transequatorial loops were part of the complex magnetic field and they formed part of the CME material, a configuration not yet included in the breakout simulations.

The February 8, 2000 halo CME event was associated with a large number of ejecta, with different trajectories and source regions. It is possible that instead of one CME bubble or loop we may have a complex structure that consists of a variety of erupting material. And even if this event was well-covered in wavelengths, $\mathrm{H} \alpha$ observations still were missing, some field of views were limited, and the image cadence of some instruments was inadequate to follow the full evolution of all the structures. Therefore, it remains to be investigated if our results can be generalized to other halo CMEs and to all types of CMEs.

Acknowledgements. We would like to thank A. Klassen for the preparation of the Tremsdorf radio spectra, and K.-L.Klein for checking the validity of the Nançay data. Ondřejov radio data are available at their web site. Yohkoh SXT and HXT data were obtained from the DARTS Yohkoh database maintained at ISAS/JAXA, Japan. TRACE is a NASA Small Explorer (SMEX) mission. The LASCO CME catalog is generated and maintained by NASA and The Catholic University of America in cooperation with the Naval Research Laboratory. SOHO is a project of international cooperation between ESA and NASA. S.P. acknowledges a travel grant from the Väisälä Foundation of the Finnish Academy of Science and Letters.

\section{References}

Antiochos, S. K. 1998, ApJ, 502, L181

Antiochos, S. K., DeVore, C. R., \& Klimchuk, J. A. 1999, ApJ, 510, 485

Aurass, H., \& Klein, K.-L. 1997, A\&AS, 123, 279

Aurass, H., Vourlidas, A., Andrews, M. D., et al. 1999, ApJ, 511, 451

Bastian, T. S., Benz, A. O., \& Gary, D. E. 1998, ARA\&A, 36, 131

Bastian, T., Pick, M., Kerdraon, A., Maia, D., \& Vourlidas, A. 2001, ApJ, 558, L65

Benz, A. O. 2003, Lecture Notes in Physics, 612, 80

Biesecker, D. A., Myers, D. C., Thompson, B. J., Hammer, D. M., \& Vourlidas, A. 2002, ApJ, 569, 1009

Brueckner, G. E., Howard, R. A., Koomen, M. J., et al. 1995, Sol. Phys., 162, 357

Caroubalos, C., Poquerusse, M., Bougeret, J.-L., \& Crepel, R. 1987, ApJ, 319, 503
Caroubalos, C., Maroulis, D., Patavalis, N., et al. 2000, Exp. Astron., 11,23

Caroubalos, C., Hillaris, A., Bouratzis, C., et al. 2004, A\&A, 413, 1125

Claßen, H. T., \& Aurass, H. 2002, A\&A, 384, 1098

Delaboudinière, J.-P. and 27 colleagues 1995, Sol. Phys., 162, 291

Glover, A., Harra, L. K., Matthews, S. A., \& Foley, C. A. 2003, A\&A, 400,759

Gopalswamy, N., \& Kundu, M. R. 1990, Sol. Phys., 128, 377

Handy, B. N., Acton, L. W., Kankelborg, C. C., et al. 1999, Sol. Phys., 187,229

Harra, L. K., \& Sterling, A. C. 2001, ApJ, 561, L215

Hudson, H. S., Khan, J. I., Lemen, J. R., Nitta, N. V., \& Uchida, Y. 2003, Sol. Phys., 212, 121

Jiricka, K., Karlický, M., Kepka, O., \& Tlamicha, A. 1993, Sol. Phys., 147, 203

Kahler, S. W. 1992, ARA\&A, 30, 113

Kosugi, T., Masuda, S., Makishima, K., et al. 1991, Sol. Phys., 136, 17

Kerdraon, A., \& Delouis, J.-M. 1997, in Coronal Physics from Radio and Space Observations, ed. G. Trottet (Springer), 192

Khan, J. I., \& Hudson, H. S. 2000, GeoRL, 27, 1083

Klein, K.-L., Khan, J. I., Vilmer, N., Delouis, J.-M., \& Aurass, H. 1999 A\&A, 346, L53

Klein, K.-L., \& Mouradian, Z. 2002, A\&A, 381, 683

Klein, K.-L., Schwartz, R. A., McTiernan, J. M., et al. 2003, A\&A, 409,317

Kundu, M. R. 1965, Solar Radio Astronomy, Interscience

Leblanc, Y., Dulk, G. A., Vourlidas, A., \& Bougeret, J.-L. 2001, JGR, 106,25301

MacNeice, P., Antiochos, S. K., Phillips, A., et al. 2004, ApJ, 614, 1028

Maia, D., Aulanier, G., Wang, S. J., et al. 2003, A\&A, 405, 313

Maia, D., Pick, M., Kerdraon, A., et al. 1998, Sol. Phys., 181, 121

Maia, D., Pick, M., Vourlidas, A., \& Howard, R. 2000, ApJ, 528, L49

Maia, D., Vourlidas, A., Pick, M., et al. 1999, JGR, 104, 12507

Mann, G., Aurass, H., Voigt, W., \& Paschke, J. 1992, ESA-Journal SP-348, 129

Manoharan, P. K., \& Kundu, M. R. 2003, ApJ, 592, 597

Pohjolainen, S., Maia, D., Pick, M., et al. 2001, ApJ, 556, 421

Scherrer, P. H., Bogart, R. S., Bush, R. I., et al. 1995, Sol. Phys., 162, 129

Sterling, A. C., \& Hudson, H. S. 1997, ApJ, 491, L55

Sterling, A. C., \& Moore, R. L. 2004, ApJ, 602, 1024

Thompson, B. J., Cliver, E. W., Nitta, N., Delannée, C., \& Delaboudinière, J. P. 2000, GeoRL, 27, 1431

Tsuneta, S., Acton, L., Bruner, M., et al. 1991, Sol. Phys., 136, 37

Vlahos, L., \& Raoult, A. 1995, A\&A, 296, 844

Wang, T., Yan, Y., Wang, J., Kurokawa, H., \& Shibata, K. 2002, ApJ, 572,580

Warmuth, A., Vrsnak, B., Aurass, H., \& Hanslmeier, A. 2001, ApJ, 560, L105

Wild, J. P., \& Smerd, S. F. 1972, ARA\&A, 10, 159

Zarro, D. M., Sterling, A. C., Thompson, B. J., Hudson, H. S., \& Nitta, N. 1999, ApJ, 520, L139

Zhang, M., \& Golub, L. 2003, ApJ, 595, 1251 